\title{
On Geometric Graphs with No Two Edges in Convex Position*
}

\author{
M. Katchalski and H. Last \\ Department of Mathematics, Technion-Israel Institute of Technology, \\ Haifa 32000, Israel \\ meirk@techunix.technion.ac.il
}

Communicated by János Pach

\begin{abstract}
A geometric graph is a graph $G=G(V, E)$ drawn in the plane, where its vertex set $V$ is a set of points in general position and its edge set $E$ consists of straight segments whose endpoints belong to $V$. Two edges of a geometric graph are in convex position if they are disjoint edges of a convex quadrilateral. It is proved here that a geometric graph with $n$ vertices and no edges in convex position contains at most $2 n-1$ edges. This almost solves a conjecture of Kupitz. The proof relies on a projection method (which may have other applications) and on a simple result of Davenport-Schinzel sequences of order 2.
\end{abstract}

\section{Introduction}

A geometric graph is a graph $G=G(V, E)$ drawn in the plane whose vertex set $V$ consists of points in general position (i.e., no three are collinear) and whose edge set $E$ consists of straight segments whose endpoints belong to $V$. Consult [4] for recent results on geometric graphs. Two segments are in convex position if they are disjoint edges of a convex quadrilateral. A geometric graph is called proper if it has two edges in convex position. Otherwise, it is called improper.

The systematic study of geometric graphs was initiated by Kupitz and Perles [1]. Kupitz [2] constructed improper graphs with $n$ vertices and $2 n-2$ edges for $n \geq 4$. (See Fig. 1 for an improper geometric graph on 7 vertices and 12 edges.)

\footnotetext{
* Research of the first author was supported by the Fund for the Promotion of Research at the Technion and by the M. and M. L. Bank Mathematics Research Fund.
} 


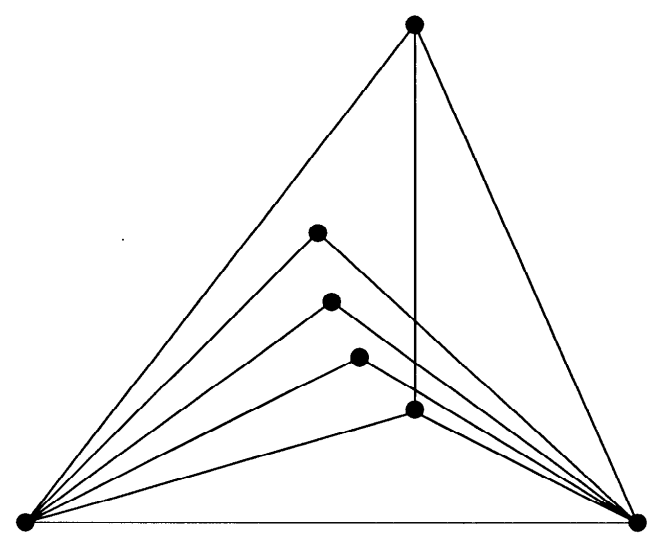

Fig. 1. An improper geometric graph on 7 vertices and 12 edges.

He made the following conjecture:

Kupitz Conjecture. An improper geometric graph with $n$ vertices has at most $2 n-2$ edges.

Here the conjecture is almost solved:

Theorem. An improper geometric graph with $n$ vertices has at most $2 n-1$ edges.

This result has appeared in [3]. The proof relies on a projection method and on a simple property of Davenport-Schinzel sequences of order 2.

The second section deals with definitions and two lemmas, followed by the proof of the theorem in the third section.

\section{Definitions and Two Lemmas}

For distinct points $x$ and $y$ let $x y$ denote the segment with endpoints $x$ and $y$, let $\ell(x, y)$ denote the line through these points, and let $\overrightarrow{x y}$ denote the ray (half-line) with apex $x$ and containing $y$. An edge $x y$ of a geometric graph is said to be to the right (left) of edge $x z$ if the ray $\overrightarrow{x y}$ is obtained from the ray $\overrightarrow{x z}$ by a clockwise (counterclockwise) rotation about $x$ by a positive angle less than $\pi$. If there is no edge incident to $x$ to the right (left) of $x y$, then $x y$ is called the rightmost (leftmost) edge of $x$. If $x y$ is not the rightmost or the leftmost edge of $x$, then it is called an interior edge of $x$.

A circular sequence is a sequence whose first and last term are considered adjacent. A circular sequence from a set of $n$ symbols shall be called a circular Davenport-Schinzel sequence of order 2 if no two adjacent terms are identical and if it does not contain a circular subsequence of type $a b a b$.

For more advanced results on Davenport-Schinzel sequences (which are not needed here), applications, etc., we may consult [5]. We shall need a known upper bound on the 
length of such circular sequences of order 2 which is easily proved by induction on $n$, the number of symbols, see [5].

Lemma 1. The length of a circular Davenport-Schinzel sequence of order 2 on $n$ symbols for $n \geq 2$ is at most $2 n-2$.

A convex curve is the boundary of a compact convex planar set with nonempty interior. The next technical lemma is essential to the proof of the theorem.

Lemma 2. Let $A_{i}, A_{j}, A_{k}, A_{l}$ be four points appearing in this order on a convex curve $\gamma$. Let $P, Q$ be two points inside $\gamma$.

Consider the four (closed) segments

$$
P A_{i}, Q A_{j}, P A_{k}, Q A_{\ell}
$$

and assume that among them:

there is no segment s such that s contains only one of the points $P, Q$ and the line supporting s contains both of them.

Then two of the four segments are in convex position.

Proof. Observe that:

If points $A, B, C, D$ in general position lie in this order on a convex curve, then the segments $A B$ and $C D$ are in convex position.

Let $\ell=\ell(P, Q)$ and let $l^{+}$and $l^{-}$be two half-planes such that $l^{+} \cap l^{-}=\ell$. Let $\gamma_{1}\left(\gamma_{2}\right)$ be the boundary of the convex hull of $l^{+} \cap \gamma$ (of $l^{-} \cap \gamma$ ). It is easy to check, using (2) and (3) that if one of the four points $A_{i}, A_{j}, A_{k}, A_{\ell}$ lies on $\ell$, then two of the segments are in convex position. Assume therefore without loss of generality that either

$$
A_{i}, A_{j} \text {, and } A_{k} \text { lie in the interior of } l^{+}
$$

or

$$
A_{i} \text { and } A_{j} \text { lie in the interior of } l^{+}
$$

and

$$
A_{k} \text { and } A_{\ell} \text { lie in the interior of } l^{-} .
$$

In case (4), by (3) applied to $\gamma_{1}$, either $P A_{i}$ and $Q A_{j}$ are in convex position or $P A_{k}$ and $Q A_{j}$ are in convex position.

In case (5) if $P A_{i}, Q A_{j}$ are not in convex position and $P A_{k}, Q A_{\ell}$ are not in convex position, then by (3) the order of the points on $\gamma$ is $A_{i}, A_{j}, A_{\ell}, A_{k}$, a contradiction. 


\section{Proof of the Theorem}

Let $v_{1}, \ldots, v_{n}$ be the vertices of $G$ and $e$ the number of its edges. Assume that $G$ is improper with $e \geq 1$. Let $C$ be a circle containing $v_{1}, \ldots, v_{n}$ in its interior. For any two vertices $v_{i}$ and $v_{j}$ joined by an edge $v_{i} v_{j}$ define two points on $C$ :

$$
\alpha_{i j}={\overrightarrow{v_{i}}}_{j} \cap C \quad \text { and } \quad \alpha_{j i}={\overrightarrow{v_{j}}}_{i} \cap C .
$$

Arrange the $2 e$ points on $C$ in a circular sequence according to the order of their appearance on $C$. Let $D(G)$ be the circular sequence thus obtained.

Color the points of $D(G)$ with $n$ colors such that

$$
\alpha_{i j} \text { receives the color } i \text {. }
$$

Point $\alpha_{i j}$ has a dark color $i$ if $v_{i} v_{j}$ is an interior edge of $v_{j}$. Otherwise, $\alpha_{i j}$ has a light color $i$. Divide the sequence $D(G)$ into $\operatorname{arcs}$ where an arc is a maximal subsequence of consecutive points of $D(G)$ having the same color $i$. Note that a dark $i$ and a light $i$ may belong to the same arc.

The circular sequence obtained from $D(G)$ by contracting each arc to one of its points and then replacing the point by its color $i$ is called the pattern sequence of $G$, or $P S(G)$. See Fig. 2. for a geometric graph with four vertices and the corresponding sequences $D(G)$ and $P S(G)$. Behind each point $\alpha_{i j}$ is shown its color with a superscript $d$ if it is dark or $\ell$ if it is light.

Here $D(G)=\left(\alpha_{41}, \alpha_{42}, \alpha_{43}, \alpha_{23}, \alpha_{12}, \alpha_{14}, \alpha_{24}, \alpha_{34}, \alpha_{32}, \alpha_{21}\right)$. The arcs of $G$ are $\left(\alpha_{41}, \alpha_{42}, \alpha_{43}\right),\left(\alpha_{23}\right),\left(\alpha_{12}, \alpha_{14}\right),\left(\alpha_{24}\right),\left(\alpha_{34}, \alpha_{32}\right),\left(\alpha_{21}\right)$. The pattern sequence is $\operatorname{PS}(G)$ $=(4,2,1,2,3,2)$.

We need the following two assertions:

Lemma 3. $P S(G)$ is a circular Davenport-Schinzel sequence of order 2.

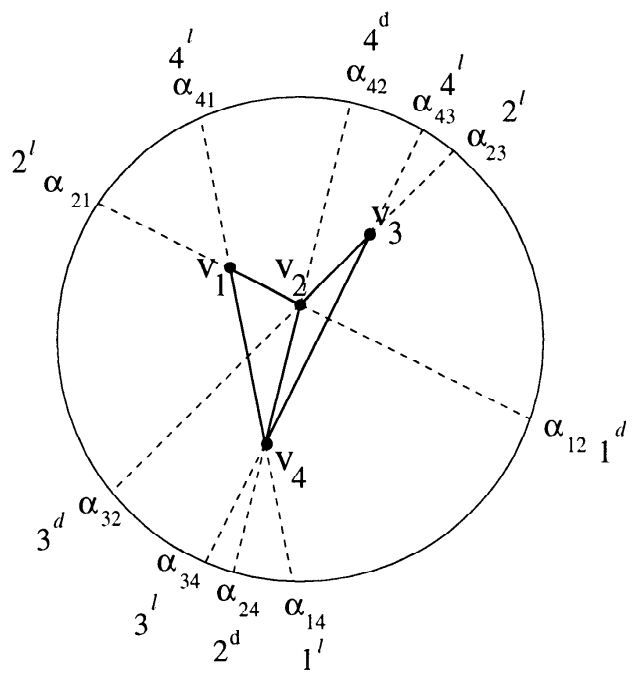

Fig. 2. A geometric graph on four vertices and the derived sequence. 
Lemma 4. An arc of $D(G)$ contains at most one point with dark color.

We have

$$
|D(G)|=2 e=\sharp \text { of light colored points }+\sharp \text { of dark colored points. }
$$

Each vertex of $G$ has at most one rightmost edge and at most one leftmost edge incident to it, so that the number of light colored points in $D(G)$ is bounded by $2 n$. By Lemmas 1 , 3 , and 4,

$$
\sharp \text { of dark colored points } \leq|P S(G)| \leq 2 n-2 .
$$

Substitute all of the above in (6) to obtain

$$
e \leq 2 n-1
$$

It remains to prove Lemmas 3 and 4.

Proof of Lemma 3. If $P S(G)$ is not a circular Davenport-Schinzel sequence of order 2 , then there are four points, $\alpha_{a u_{1}}, \alpha_{b u_{2}}, \alpha_{a u_{3}}, \alpha_{b u_{4}}$ appearing in that order on $C$. Since $v_{1}, \ldots, v_{n}$ are in general position there are no two disjoint segments $v_{x} v_{y}, v_{z} v_{t}$ such that a line through one of them contains an endpoint of the other. Therefore by Lemma 2, two of the segments

$$
v_{a} \alpha_{a u_{1}}, v_{b} \alpha_{b u_{2}}, v_{a} \alpha_{a u_{3}}, v_{b} \alpha_{b u_{4}}
$$

are in convex position. Since every edge $v_{x} v_{y}$ is contained in the segment $v_{x} \alpha_{x y}$, two of the segments

$$
v_{a} v_{u_{1}}, v_{b} v_{u_{2}}, v_{a} v_{u_{3}}, v_{b} v_{u_{4}}
$$

are in convex position, a contradiction.

Proof of Lemma 4. Suppose that the points $\alpha_{a b}, \alpha_{a c}$ belong to the same arc and are dark colored. Assume without loss of generality that $v_{a} v_{c}$ is to the right of $v_{a} v_{b}$. The edges $v_{a} v_{b}, v_{a} v_{c}$ are interior edges of $v_{b}$ and $v_{c}$, respectively. So let $v_{b} v_{x}$ be to the right of $v_{b} v_{a}$ and let $v_{c} v_{y}$ be to the left of $v_{c} v_{a}$. Let $s t$ be the chord of $C$ that contains $v_{b} v_{c}$, so that $v_{b}$ lies in $v_{c} s$ and $v_{c}$ in $v_{b} t$. Let $\vec{r}$ be the ray with apex $v_{b}$ and parallel to $\vec{v}_{c} \vec{v}_{a}$.

Let $\alpha_{1}, \alpha_{2}, \alpha_{3}, \alpha_{4}$ be the following angles:

$$
\begin{aligned}
\alpha_{1} & =\operatorname{conv}\left(\overrightarrow{v_{b} \alpha_{b a}} \cup \vec{r}\right), \\
\alpha_{3} & =\operatorname{conv}\left(\overrightarrow{v_{b} s} \cup \overrightarrow{v_{b} \alpha_{a b}}\right)
\end{aligned} \quad \text { and } \quad \begin{array}{ll}
\alpha_{2} & =\operatorname{conv}\left(\vec{r} \cup \overrightarrow{v_{b} s}\right), \\
\alpha_{4} & =\operatorname{conv}\left(\overrightarrow{v_{c} t} \cup \overrightarrow{v_{c} \alpha_{a c}}\right) .
\end{array}
$$

See Fig. 3.

Since $v_{b} v_{x}$ is to the right of $v_{b} v_{a}, v_{x}$ must lie in at least one of the angles $\alpha_{1}$ or $\alpha_{2}$ or $\alpha_{3}$. If $v_{x} \in \alpha_{1}$, then the points $\alpha_{a c}, \alpha_{x b}, \alpha_{a b}, \alpha_{b x}$ are in that order on $C$. Therefore $\alpha_{a b}, \alpha_{a c}$ are not on the same arc, a contradiction. If $v_{x} \in \alpha_{2}$, then $v_{b} v_{x}$ and $v_{c} v_{a}$ are in convex position, a contradiction.

Therefore $v_{b} v_{x}$ is in $\alpha_{3}$ and by symmetry $v_{c} v_{y}$ is in $\alpha_{4}$, implying $v_{b} v_{x}$ and $v_{c} v_{y}$ are in convex position, a contradiction. 


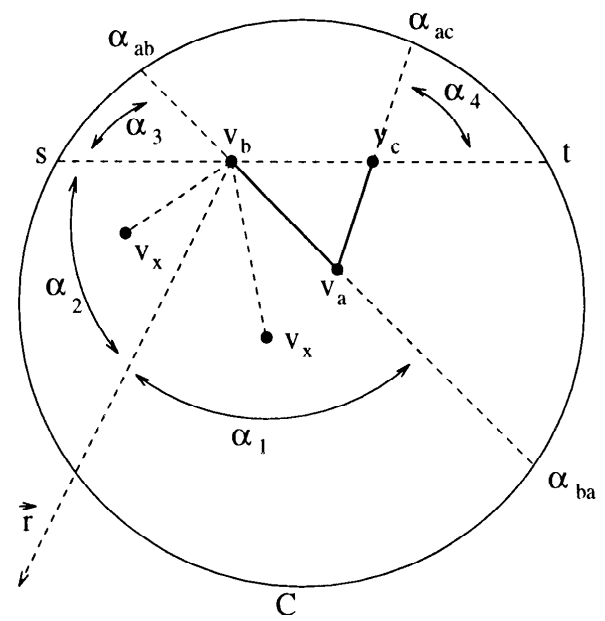

Fig. 3. Two dark points in an arc of an improper geometric graph are impossible.

The conjecture of Kupitz has been proved by Pavel Valtr, consult [6]. The major contribution of [6] is that for any fixed $k \geq 3$, any geometric graph on $n$ vertices with no $k$ edges such that any two of them are in convex position contains at most $O(n)$ edges. Valtr also proves that any geometric graph on $n$ vertices with no $k$ pairwise crossing edges contains at most $O(n \log n)$ edges.

\section{Acknowledgments}

Finally, the authors thank the referees and Pavel Valtr for their corrections and helpful suggestions.

\section{References}

1. Y. Kupitz, Extremal Problems in Combinatorial Geometry, Aarhus University Lecture Notes Series, vol. 53, 1979.

2. Y. Kupitz, On pairs of disjoint segments in convex position in the plane, Ann. Discrete Math., 20 (1984), 203-208.

3. H. Last, Turán type problems for geometric graphs, Ms.C. thesis, Technion-I.I.T., 1994. In Hebrew.

4. J. Pach and P. K. Agarwal, Combinatorial Geometry, Wiley Interscience, New York, 1995.

5. M. Sharir and P. K. Agarwal, Davenport-Schinzel Sequences and Their Geometric Applications, Cambridge University Press, Cambridge, 1995.

6. P. Valtr, On geometric graphs with no $k$ pairwise parallel edges, Discrete Comput. Geom., this issue, pp. 461-469.

Received December 18, 1995, and in revised form June 17, 1997. 